Ann. Génét. Sél. anim., I97I, 3 (4), 4I3-4I8.

\title{
POLYMORPHISME DE LA PHOSPHOGLUCOMUTASE (PGM) DANS L'ESPÈCE BOVINE (1)
}

\author{
M. ANSAY, R. HANSET et J. ESSER-COULON \\ Chaire de Génétique, Faculté de Médecine vétérinaire (Cureghem-Bruxelles), \\ Université de Liège
}

\section{RÉSUMÉ}

L'hétérogénéité de la phosphoglucomutase (PGM) est décrite dans l'espèce bovine. Par analogie avec les observations faites dans l'espèce humaine, l'existence de trois loci, caractérisées par une expression différente selon les tissus, paraît très probable. Le locus $\mathrm{PGM}_{1}$ est le siège d'un polymorphisme génétique, déterminé par une paire d'allèles co-dominants. Les trois génotypes correspondants sont symbolisés par $P_{G} M_{1}-A A, P G M_{1}-A B, P G M_{1}-B B$. La fréquence de l'allèle $\mathrm{PGM}_{1}$-A est estimée dans quelques races bovines.

\section{INTRODUCTION}

Ia phosphoglucomutase (E. C. 2. 7.5. I. ; $\alpha$-D-glucose-I-6 diphosphate : $\alpha$-D-glucose-I phosphate phosphotransférase ; PGM) catalyse la conversion du glucoseI-phosphate en glucose-6-phosphate.

Chez 1'Homme, cette enzyme montre, à l'analyse, une très grande hétérogénéité : les causes en sont multiples. Ainsi, trois loci distincts contrôlent la synthèse de trois types de phosphoglucomutase $\left(\mathrm{PGM}_{1}, \mathrm{PGM}_{2}, \mathrm{PGM}_{3}\right.$ : variation cistronique) différemment distribués dans les tissus. (SPENCER et al., I964 ; Hopkins et HARRIs, I965 ; Hopkinson et HARRIS, I968). En outre, il existe, à chacun de ces loci, une variation allélique. Une troisième cause d'hétérogénéité, non révélée par l'électrophorèse, est due au fait que l'enzyme peut exister sous une forme phosphorylée ou non (YANKEELOV et al., I964). Enfin, la présence pour la $\mathrm{PGM}_{1}$ surtout, d'isomères de configuration ou " conformers" est une quatrième source d'hétérogénéité (DAwson et MiTcheL, I969; DAWSON et YEAGER, I970).

(1) Travail exécuté sous les auspices de l'Institut pour l'Encouragement de la Recherche scientifique dans I'Industrie et l'Agriculture (I, R. S. I. A.). 
L'objet de ce travail est de démontrer l'existence d'un polymorphisme de la phosphoglucomutase dans l'espèce bovine. Il révèle, en outre, la présence de deux zones supplémentaires, à activité PGM et à expressivité variable suivant les tissus.

\section{MATÉRIEL ET MÉTHODES}

Le mode de préparation des extraits musculaires et leucocytaires, les méthodes d'électrophorèse ont été décrits dans une publication antérieure (ANSAY et al., 197I). Les érythrocytes lavés par du $\mathrm{NaCl}$ à $0,9 \mathrm{p}$. Ioo, sont hémolysés, après addition d'un volume égal d'eau distillée, par congélations et décongélations successives. Ils sont ensuite centrifugés à roo $000 \mathrm{~g}$.

Les gels mixtes d'acrylamide (cyanogène $4 \mathrm{I}: 5 \mathrm{p}$. 100) et de gélose ( $\mathrm{I}$ p. IOO) (URIEL, I966) se sont révélés favorables à l'étude de cette enzyme.

Selon un mécanisme non encore parfaitement élucidé, le glucose I-6 diphosphate participe à la réaction catalysée par la phosphoglucomutase.

Le glucose I-6 diphosphate est habituellement présent à l'état de traces dans le glucoseI-phosphate d'origine commerciale (Sigma ou Boehringer) et il suffit, comme tel, à la révélation d'extraits, teis les extraits musculaires, naturellement riches en PGM.

Cependant, et ceci confirme les données de Hopkinson et HarRIS (I968), l'addition de glucose-I-6-diphosphate (I,5 $\mu$ mole pour ıoo $\mu$ moles de glucose-I-phosphate) accélère considérablement la réaction et surtout, permet la mise en évidence de bandes plus discrètes, telles celles de la $\mathrm{PGM}_{2}$ des globules rouges ou de la $\mathrm{PGM}_{3}$ du placenta.

La révélation du zymogramme se réalise selon une méthode positive dont les modalités ont été déjà décrites (ANSAY et al., 1971). La composition du mélange réactionnel, exprimée en concentration finale, est la suivante :

Gélose, I p. roo.

Tris- $\mathrm{HCl}, \mathrm{pH} 7,2,70 \mathrm{mM}$.

Nicotineamide adénine dinucléotide phosphate (NADP Sigma), o, $13 \mathrm{mM}$.

Glucose I-phosphate disodique (Boehringer), $4 \mathrm{mM}$.

Glucose I-6-diphosphate, sel tétracyclohexylammonique (Boehringer), 0,05 $\mathrm{mM}$.

MTT tétrazolium (Sigma), o, 3 r. I ooo (poids/volume).

Phénazine méthosulfate (PMS) (Sigma), o,02 p. I ooo (poids/volume).

Glucose-6-phosphate déhydrogénase (Boehringer), o,7 UI/ml.

\section{RÉSULTATS}

\section{A. - Expression tissulaire}

Trois zones d'activité de la phosphoglucomutase apparaissent dans les phérogrammes de tissus bovins. Chacune de ces zones est représentée de façon caractéristique, selon le tissu.

Si la zone la plus lente $\left(\mathrm{PGM}_{1}\right)$ est prépondérante dans tous les tissus examinés, elle est la seule à se manifester, avec éventuellement des isozymes de conformation, dans le muscle squelettique ou cardiaque. C'est cette zone qui est le siège de variants génétiques (fig. I $a, b$ ).

Une deuxième bande $\left(\mathrm{PGM}_{2}\right)$ à migration anodique plus rapide et n'ayant pas montré de variants, se révèle dans les zymogrammes de foie, de rein, de globules blancs, de globules rouges et de placenta (fig. I $c$ ).

Les cotylédons placentaires d'origine fœtale ou maternelle montrent une troi- 
sième zone d'activité $\left(\mathrm{PGM}_{3}\right)$ à déplacement anodique plus rapide encore que $\mathrm{PGM}_{2}$ (fig. I $d$ ).

La présence d'isozymes de conformation est caractéristique de la zone $\mathrm{PGM}_{1}$ musculaire. Ils apparaissent le mieux à $\mathrm{pH} 8,7$ (fig. I $a$ ) et beaucoup moins bien à $\mathrm{pH} 6,7$ (fig. I $b$ ).

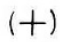

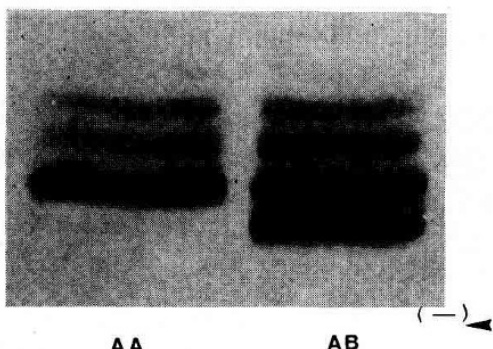

A A
$A B$

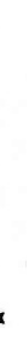

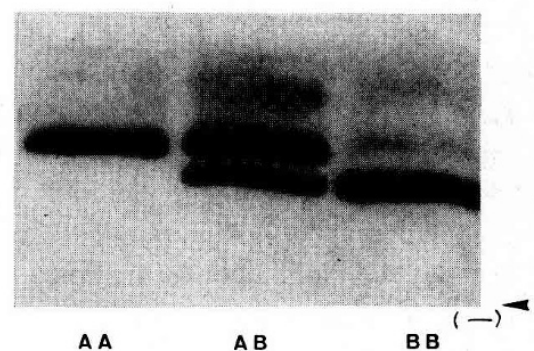

A A

b

a

\section{$(+)$}

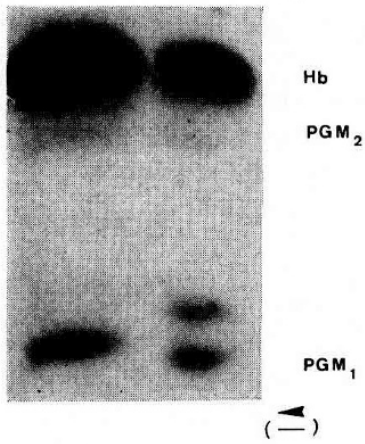

a

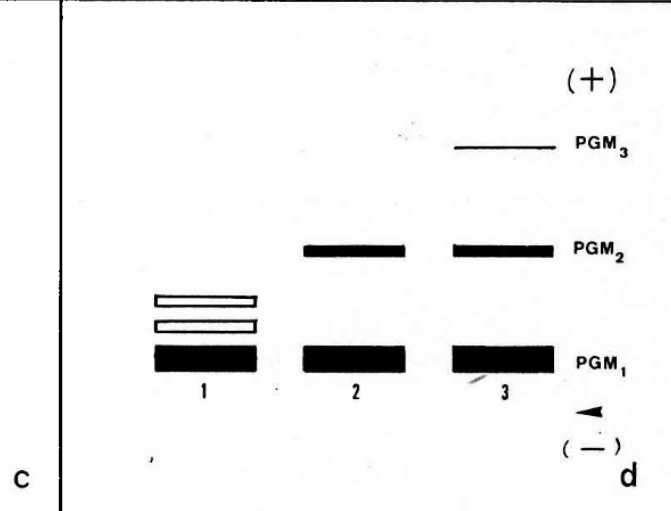

FiG. I

a) Électrophorèse en gel mixte d'acrylamide-gélose selon URIEL (1966), pH 8,75, 500 volts, 45 minutes. Extraits musculaires de phénotype $A A$ et $A B$. Démonstration des isozymes de conformation.

b) Électrophorèse en gel d'amidon (I4 p. I0o). Tampon de Shows et al. (1969), pH 6, 7, 500 volts, 1 heure. Extraits musculaires. Les 3 phénotypes sont démontrés.

c) Électrophorèse en gel d'amidon (14 p. roo). Tampon d'URIEL (I966), pH 8,75, 500 volts, 1 heure. Extraits érythrocytaires. Démonstration de 2 phénotypes de la $P G M_{1}$ et mise en évidence de la $P G M_{2}$.

d) Représentation schématique des formes enzymatiques correspondant au locus $P G_{1}$ et aux deux autres loci supposés :

I. Extrait de muscle. $\mathrm{PGM}_{1}$ et isozymes de conformation.

2. Extrait de globules blancs. $\mathrm{PGM}_{1}$ et $\mathrm{PGM}_{2}$.

3. Extrait de placenta. $\mathrm{PGM}_{1}, \mathrm{PGM}_{2}$ et $\mathrm{PGM}_{3}$.

\section{B. - Description des phénotypes ( $\left.\mathrm{PGM}_{1}\right)$}

Les zymogrammes réalisés à partir d'extraits musculaires, leucocytaires ou érythrocytaires de nombreux animaux révèlent l'existence de trois phénotypes.

Ils sont reproduits figure $\mathrm{I} b$. Deux phénotypes n'ont qu'une bande : phénotype $\mathrm{AA}$ et phénotype $\mathrm{BB}$; le troisième a deux bandes : phénotype $\mathrm{AB}$. 
Dans la figure I $a$, les phénotypes $\mathrm{AA}$ et $\mathrm{AB}$ sont représentés avec leurs sousbandes correspondant aux isozymes de conformation.

Le mélange en proportions égales d'extraits caractérisés par la présence d'une bande, lente ou rapide, révèle un zymogramme identique à celui des individus à deux bandes.

$$
\text { C. - Analyse génétique }
$$

L'existence de trois phénotypes permet de poser l'hypothèse d'une paire d'allèles $(A$ et $B)$ avec les génotypes $A A, B B$ et $A B$.

Les deux phénotypes à une bande répondraient, suivant cette hypothèse, aux génotypes $\mathrm{AA}$ ou $\mathrm{BB}$ selon que cette bande présente une vitesse de migration comparativement rapide ou lente.

Le génotype $\mathrm{AB}$ correspondrait au phénotype à 2 bandes. Dans le cas du muscle, quand les isozymes de conformation sont présents, les différences de mobilité affectent le groupe de bandes dans son ensemble.

Un taureau de race de Moyenne et Haute Belgique utilisé au Centre d'insémination artificielle de la province de Liège possédait le phénotype $\mathrm{AB}$ à deux bandes. Croisé avec 25 vaches de phénotype $\mathrm{BB}$ (génotype $\mathrm{BB}$ ), sa descendance s'est répartie en 2 classes : $I_{4}$ individus de phénotype $\mathrm{AB}$ et $\mathrm{I}$ I individus de phénotype $\mathrm{BB}$ (tabl. $\mathrm{I}$ ).

Le croisement réciproque $(\widehat{A B} \times$ 우 $\mathrm{AB})$, a donné les deux mêmes catégories d'individus (tabl. I).

Les proportions obtenues sont au total, pour ces deux derniers types de croisement, celles que l'on attend du croisement d'un hétérozygote avec des homozygotes $(I: I)$.

TABLEAU I

Les phénotypes dans la descendance de différents croisements $\left(\mathrm{PGM}_{1}\right)$

\begin{tabular}{|c|c|c|c|c|}
\hline \multicolumn{2}{|c|}{ Phénotypes parentaux } & \multicolumn{3}{|c|}{ Phénotypes des descendants } \\
\hline$\sigma$ & 우 & $\mathrm{AA}$ & $\mathrm{AB}$ & $\mathrm{BB}$ \\
\hline $\mathrm{AB}$ & BB & - & 14 & 11 \\
\hline BB & $\mathrm{AB}$ & - & 1 & 4 \\
\hline BB & BB & - & - & 19 \\
\hline
\end{tabular}

Le croisement $ð \mathrm{BB} \times \subsetneq \mathrm{BB}$ n'a donné que des descendants $\mathrm{BB}$ (tabl. I).

Ces résultats sont compatibles avec l'hypothèse de deux allèles co-dominants pour lesquels on peut proposer la symbolisation suivante : $\mathrm{PGM}_{1}-\mathrm{A}$, pour l'allèle déterminant la bande la plus rapide, $\mathrm{PGM}_{1}-\mathrm{B}$, pour l'allèle, plus commun, déterminant la bande lente.

\section{D. - Distribution raciale}

La fréquence de l'allèle $\mathrm{PGM}_{1}-\mathrm{A}$, l'allèle le moins fréquent, a été estimée dans quatre races bovines, exploitées en Belgique. Les résultats figurent dans le tableau 2. 
La fréquence du gène $\mathrm{PGM}_{1}-\mathrm{A}$ est très élevée, dans l'échantillon de vaches Jersey. Cette race n'est représentée, en Belgique, que par un nombre restreint d'individus. Dès lors, la fréquence obtenue n'est pas nécessairement représentative de la race Jersey, en général.

TABLEAU 2

La fréquence de l'allèle $P G M_{1}-A$ dans quelques races bovines

\begin{tabular}{l|c|c|c}
\hline \multicolumn{1}{c|}{ Race } & $\begin{array}{c}\text { Nombre } \\
\text { d'animaux } \\
\text { testés }\end{array}$ & $\begin{array}{c}\text { Fréquence } \\
\text { du gène } \\
(\%)\end{array}$ & $\begin{array}{c}\text { Intervalle de } \\
\text { confiance 95 \% }\end{array}$ \\
\hline Moyenne et Haute Belgique . & 148 & 7,4 & $4,8-11,0$ \\
Pie-Noire (Hollandaise) ... & 101 & 5,9 & $3,1-10,5$ \\
Pie-Rouge (MRY) ........ & 94 & 7,4 & $4,2-12,3$ \\
Jersey ........................ & 27 & 31,5 & $19,1-45,0$ \\
\hline \hline
\end{tabular}

Pour les trois autres races envisagées, Moyenne et Haute Belgique, Pie Noire, Pie Rouge (MRY), la fréquence du gène est, en moyenne, de 7 p. Ioo (intervalle de confiance 95 p. IOO : $5, \mathrm{I}-9,2)$.

\section{DISCUSSION}

L'analogie que présente la phosphoglucomutase d'origine bovine avec la phosphoglucomutase humaine est frappante.

Trois loci différents et indépendants y ont été décrits par SPENCER et al. (I964); Hopkinson et HARRIS (I965) ; Hopkinson et HARris (I968). Ils diffèrent par l'inégalité de leur expression tissulaire, par leur vitesse de migration électrophorétique et sont tous trois l'objet d'une variation allélique.

La PGM d'origine bovine, présente à l'électrophorèse divers isozymes dont l'expression tissulaire est semblable à celle de la PGM humaine ; l'ordre de migration dans le champ électrophorétique est semblable dans les deux espèces. Cependant, si le locus $\mathrm{PGM}_{1}$ a pu être sûrement identifié par une variation allélique, il n'en est pas de même pour les deux autres qui jusqu'ici, n'ont pas révélé de polymorphisme.

Chez la souris, SHows et al. (I969) ont décrit un polymorphisme de la PGM. Les deux zones majeures habituellement présentes sont sous le contrôle de deux loci génétiques $\mathrm{PGM}_{1}$ et $\mathrm{PGM}_{2}$.

Le phénotype hétérozygote bovin se manifeste par la présence de deux bandes seulement : cela le différencie de $1 \mathrm{a} \mathrm{MOR}_{1}$ bovine (ANSAy et al., I97I) qui présentait trois bandes à l'état hétérozygote. Ce fait est, de plus, en accord avec les travaux de Joshr et al. (I967) indiquant que la phosphoglucomutase est constituée d'une seule chaîne polypeptidique. 


\section{REMERCIEMENTS}

Nous adressons nos remerciements au docteur J. Marcourt, directeur du Centre I. A. de la Province de Liège pour sa collaboration dans la récolte du matériel utile à l'analyse génétique.

Nous remercions également MM. F. Grosclaude et J.-J. LAUVERGNE pour leurs suggestions concernant la formulation de nos conclusions.

\section{SUMMARY}

POLYMORPHISM OF BOVINE PHOSPHOGLUCOMUTASE (PGM)

The heterogeneity of bovine phosphoglucomutase is described. In analogy with the human species, the existence of three loci appears very likely. Genetic variants occur in locus PGM . They define a locus with two co-dominant alleles (suggested symbols : $\mathrm{PGM}_{1}-\mathrm{AA}, \mathrm{PGM}_{1}-\mathrm{AB}$, $\left.\mathrm{PGM}_{1}-\mathrm{BB}\right)$. The frequency of the allele $\mathrm{PGM}_{1}-\mathrm{A}$ is estimated in four breeds of cattle.

\section{RÉFÉRENCES BIBLIOGRAPHIQUES}

Ansay M., Hanset R., Esser-Coulon J., I97r. La malate déshydrogénase mitochondriale; variants électrophorétiques de nature héréditaire dans l'espèce bovine. Ann. Génét. Sél. anim. (sous presse).

Dawson D. M., Mitchell A., rg69. The isoenzymes of phosphoglucomutase. Biochemistry, 8, 6o9614.

Dawson D. M., Jaeger S., r97o. Heterogeneity of phosphoglucomutase. Biochem. Genet., 4, r-ro. HARRIS H., 1970. The principles of human biochemical genetics. North Holland Publishing Company. Amsterdam-London.

Hopkinson D. A., Harris H., 1965. Evidence for a second "structural " locus determining human phosphoglucomutase. Nature, 208, 4I0-4I2.

Hopkinson D. A., Harris H., r968. A third phosphoglucomutase locus in man. Ann. Hum. Genet. $31,359-367$.

Joshi J. G., Hooper J., Kuwaki T., Sakurada T., Swanson J. R., Handler P., I967. Phosphoglucomutase, V, Multiple forms of phosphoglucomutase. Proc. Natl. Acad. Sci., U. S. A., 57, I482-1489.

Shows T. B., Ruddle F. H., Roderick T. H., 1969. Phosphoglucomutase electrophoretic variants in the mouse. Biochem. Genet., 3, 25-35.

SPENCER N., Hopkinson D. A., HARRis H., I964. Phosphoglucomutase polymorphism in man. Nature, 204, 742-745.

Uriel J., I966. Méthode d'électrophorèse dans des gels d'acrylamide-agarose. Bull. Soc. Chim. Biol,. 48, 969-982.

Yankeelov J. A. Jr., Horton H. R., Koshland D. E. Jr, I964. A chromatographic study of phosphoglucomutase : separation of Phospho- and dephospho-enzyme forms. Biochemistry, 3, 349-355. 